\title{
Determinants of survival in patients with bladder cancer undergoing radical cystectomy: The impact of serum creatinine level
}

\author{
Paweł Hackemer ${ }^{1, A-D}$, Bartosz Małkiewicz ${ }^{1, A, D, E}$, Fryderyk Menzel ${ }^{2, B-D}$, \\ Aleksandra Drabik ${ }^{3, C D}, K^{2} z y s z t o f$ Tupikowski ${ }^{4, C}$, Romuald Zdrojowy ${ }^{1, E, F}$ \\ ${ }^{1}$ Department of Urology and Oncologic Urology, Wroclaw Medical University, Poland \\ ${ }^{2}$ Department of Hygiene, Wroclaw Medical University, Poland \\ ${ }^{3}$ Department of Endocrinology, Diabetes and Isotope Therapy, Wroclaw Medical University, Poland \\ ${ }^{4}$ Department of Urology, Lower Silesian Oncology Center, Wrocław, Poland \\ A - research concept and design; $\mathrm{B}$ - collection and/or assembly of data; $\mathrm{C}$ - data analysis and interpretation; \\ $\mathrm{D}$ - writing the article; $\mathrm{E}$ - critical revision of the article; $\mathrm{F}$ - final approval of the article
}

\section{Address for correspondence}

Bartosz Małkiewicz

E-mail: bartosz.malkiewicz@umed.wroc.pl

Funding sources

None declared

Conflict of interest

None declared

Received on October 19, 2020

Reviewed on October 21, 2020

Accepted on November 18, 2020

Cite as

Hackemer P, Małkiewicz B, Menzel F, Drabik A, Tupikowski K, Zdrojowy R. Determinants of survival in patients with bladder cancer undergoing radical cystectomy: The impact of serum creatinine level. Adv Clin Exp Med. 2021;30(1):77-82. doi:10.17219/acem/130597

DOI

DOl: 10.17219/acem/130597

Copyright

Copyright by Author(s)

This is an article distributed under the terms of the

Creative Commons Attribution 3.0 Unported (CC BY 3.0)

(https://creativecommons.org/licenses/by/3.0/)

\begin{abstract}
Background. Bladder cancer is one of the most common cancers in Europe and is mostly found in men. Cystectomy is the treatment for invasive tumors that infiltrate the muscle of the bladder. This procedure is associated with a large number of complications. Eligibility for surgical treatment is important, because surgery may shorten the patient's life. The main prognostic factor is the severity of the disease, but less specific factors can be very helpful in selecting the form of treatment.
\end{abstract}

Objectives. To identify and analyze factors affecting significantly the survival in patients undergoing radical cystectomy $(R C)$.

Material and methods. A retrospective analysis of a group of 129 patients treated at the Department of Urology and Urological Oncology of University Hospital in Wrocław (Poland) was carried out. Furthermore, information about the results of laboratory tests from the medical records (blood count, creatinine concentration, etc.) was obtained. The follow-up was performed twice during the postoperative period. The Kaplan-Meier method was used to determine overall survival (OS) curves and statistical significance was assessed using log-rank test.

Results. A statistically significant correlation between preoperative serum creatinine level and $0 S$ was found. The OS was significantly shorter in patients with higher serum creatinine levels (log-rank test; $p=0.002$ ). The patients were divided into different groups to exclude the relationship between the elevated creatinine concentration and the local disease advancement. The analysis was performed in patients with and without hydronephrosis. In both groups, creatinine levels above the acceptable range were associated with a shorter survival.

Conclusions. Due to the high perioperative mortality, mainly in patients with advanced disease, it is necessary to develop the qualification process for surgical treatment. The awareness of the relationship between elevated creatinine levels and worse prognosis seems to be helpful.

Key words: survival, creatinine, hydronephrosis, cystectomy, urinary bladder neoplasms 


\section{Introduction}

Bladder cancer is a major health problem in Poland; according to European Association of Urology, it is the $7^{\text {th }}$ most commonly diagnosed cancer in male population worldwide and $5^{\text {th }}$ in Europe. Radical cystectomy (RC) is a standard treatment for muscle-invasive bladder cancer (MIBC); however, it is also recommended for patients with non-muscle-invasive tumor who are at the highest risk of progression - including high risk and recurrent nonmuscle-invasive tumours, BCG-refractory, BCG-relapsing and BCG-unresponsive, T1G3 tumours. Although there are number of prognostic factors affecting survival in patients undergoing RC such as tumor grade, tumor stage, preoperative uremia, and lymph node involvement and renal insufficiency, further reliable predictors are needed. Serum creatinine level is one of routine serum biomarkers commonly used in clinical practice associated with renal function. We aimed to determine the impact of serum creatinine levels and hydronephrosis on survival in patients who underwent RC.

\section{Material and methods}

Medical records of 143 patients from the Department of Urology and Urological Oncology of University Hospital in Wrocław (Poland), who underwent RC between February 2011 and February 2014, were evaluated retrospectively. Indications for $\mathrm{RC}$ in the presented group were MIBC (T2-T4), refractory superficial high-grade tumors as well as palliative reasons. Ten patients who had incomplete medical data were excluded. Four other patients were eliminated from the study since they could not be reached during the postoperative period. The final group consisted of 129 patients.

Table 1 describes the characteristics of 129 patients studied. The mean age was 65.5 years (range: $35-83$ years, median: 65 years) and the majority $(105 ; 82 \%)$ of patients were male. As for the stage, we observed that $79.8 \%$ of the patients presented MIBC (T2-T4); involvement of lymph nodes (N1-N3) was observed in $34.9 \%$ of the patients and hydronephrosis in 44 patients (34.1\%).

Our regular preoperative blood evaluation included serum creatinine level. Blood samples were drawn on the $1^{\text {st }}$ day of hospitalization, 1-3 days prior to RC. The normal range serum creatinine level in our laboratory was between $0.8 \mathrm{mg} / \mathrm{dL}$ and $1.3 \mathrm{mg} / \mathrm{dL}$ in men and between $0.7 \mathrm{mg} / \mathrm{dL}$ and $1.1 \mathrm{mg} / \mathrm{dL}$ in women. Therefore, the value of $1.3 \mathrm{mg} / \mathrm{dL}$ in men and $1.1 \mathrm{mg} / \mathrm{dL}$ in women was chosen as a cutoff between patients with high and low serum creatinine level.

Hydronephrosis was assessed using the ultrasound scan. In each patient, an ultrasound imaging of urinary tract (including kidneys, ureters and urinary bladder) was performed 1-3 days before the surgery. A visible
Table 1. Characteristics of patients studied

\begin{tabular}{|c|c|}
\hline Parameter & n (\%) \\
\hline \multicolumn{2}{|c|}{ Age [years] } \\
\hline Mean/median & $65.5 \pm 10.4$ \\
\hline Variation & $35-83$ \\
\hline \multicolumn{2}{|c|}{ Sex } \\
\hline Male & $105(81.4)$ \\
\hline Female & $24(18.6)$ \\
\hline \multicolumn{2}{|c|}{ Tumor stage } \\
\hline TO & $4(3.1)$ \\
\hline $\mathrm{Ta}$ & $1(0.8)$ \\
\hline Tis & $14(10.9)$ \\
\hline $\mathrm{T} 1$ & $7(5.4)$ \\
\hline $\mathrm{T} 2$ & $24(18.6)$ \\
\hline T3 & $35(27.1)$ \\
\hline $\mathrm{T} 4$ & $44(34.1)$ \\
\hline \multicolumn{2}{|c|}{ Lymph nodes involvement } \\
\hline NO & $80(62.0)$ \\
\hline N1 & $11(8.5)$ \\
\hline N2 & $32(24.8)$ \\
\hline N3 & $2(1.6)$ \\
\hline $\mathrm{Nx}$ & $4(3.1)$ \\
\hline \multicolumn{2}{|c|}{ Hydronephrosis } \\
\hline Yes & $44(34.1)$ \\
\hline No & $85(65.9)$ \\
\hline
\end{tabular}

dilation of the renal pelvis in one or both kidneys, regardless of the stage of advancement, was considered as hydronephrosis.

The follow-up was performed twice during the postoperative period. In June 2014, patients were contacted by telephone. Traditional mail was sent to patients who could not be reached by telephone. For patients who could not be contacted neither by telephone nor by traditional mail, the information whether the person is alive was received from civil registry office in November 2014. In May 2016, a $2^{\text {nd }}$ follow-up was performed by telephone and regular mail. Median follow-up time was $30.3 \pm 13.4$ months (mean: 30.1 months, min. 5.2 months, max. 60.5 months). In this study, a median follow-up is the time median between a surgery and the time when information about the patient was gathered.

Due to the retrospective nature of the study, it was not possible to assess cancer-specific survival. Information on the cause of death was obtained mainly from relatives.

The data was analyzed using STATISTICA v. 12.5 software (StatSoft Inc., Tulsa, USA). The Kaplan-Meier method was used to determine overall survival (OS) curves and statistical significance was assessed using log-rank test. Univariate analysis was performed using KaplanMeier method. Multivariate analysis was performed using 
the Cox proportional hazard regression model with a confidence interval of $95 \%$ (95\% CI). The value of $\mathrm{p}<0.05$ was considered statistically significant.

\section{Results}

The mean preoperative serum creatinine level in studied group was $1.29 \mathrm{mg} / \mathrm{dL}(0.66-2.66 \mathrm{mg} / \mathrm{dL})$. In the univariate analysis using Kaplan-Meier method, a statistically significant correlation between preoperative serum creatinine level and OS was found. The OS was significantly shorter in patients with higher serum creatinine levels (log-rank test; $\mathrm{p}=0.002$; Fig. 1 ).

Table 2 shows OS time in patients classified by serum creatinine levels. Both 90-day overall survival $(89.3 \%$ in the high-creatinine group and $94.5 \%$ in the low-creatinine group) and one-year overall survival (58.6\% and $81.2 \%$, respectively) were significantly shorter in patients with elevated creatinine levels.

The univariate analysis was performed to identify the factors affecting OS (Table 3). In the multivariate

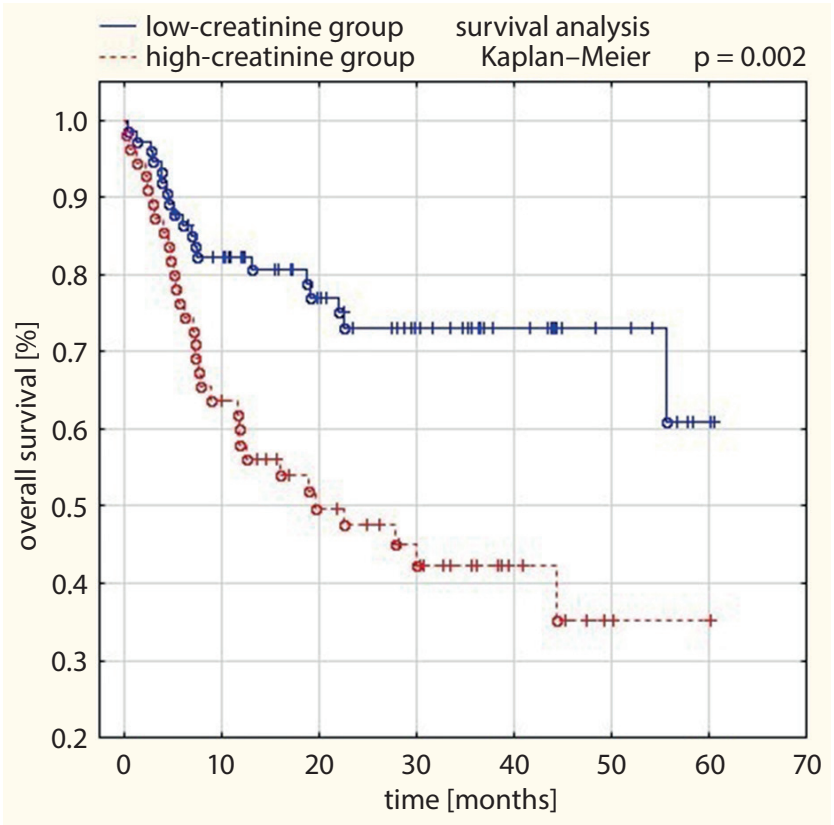

Fig. 1. Comparison of Kaplan-Meier survival curves between low and high creatinine groups

Table 2. Overall survival (OS) in patients classified by serum creatinine level

\begin{tabular}{|l|c|c|}
\hline \multicolumn{1}{|c|}{ Survival } & Low-creatinine group & High-creatinine group \\
\hline 30-day & $98.70 \%$ & $96.40 \%$ \\
\hline 90-day & $94.50 \%$ & $89.30 \%$ \\
\hline One-year & $81.20 \%$ & $58.60 \%$ \\
\hline Two-year & $72.30 \%$ & $48.50 \%$ \\
\hline Three-year & $68.50 \%$ & $45.60 \%$ \\
\hline
\end{tabular}

Table 3. Results of Cox regression to determine the association with overall survival (OS; univariate analysis)

\begin{tabular}{|l|l|l|l|}
\hline \multicolumn{1}{|c|}{ Parameter } & HR & $95 \% \mathrm{Cl}$ & p-value \\
\hline Age & 1.04 & {$[1.01-1.07]$} & 0.005 \\
\hline RBC & 0.29 & {$[0.18-0.47]$} & 0.000 \\
\hline WBC & 1.02 & {$[0.97-1.09]$} & 0.399 \\
\hline PLT & 1.00 & {$[1.00-1.01]$} & 0.003 \\
\hline HGB & 0.63 & {$[0.54-0.74]$} & 0.000 \\
\hline Na+ & 0.92 & {$[0.83-1.01]$} & 0.092 \\
\hline K+ & 2.18 & {$[1.06-4.50]$} & 0.035 \\
\hline Blood loss & 1.00 & {$[1.00-1.00]$} & 0.008 \\
\hline Creatinine & 3.18 & {$[1.80-5.62]$} & 0.000 \\
\hline GFR & 0.98 & {$[0.97-0.99]$} & 0.004 \\
\hline T & 1.71 & {$[1.22-2.41]$} & 0.002 \\
\hline N & 1.41 & {$[1.07-1.85]$} & 0.015 \\
\hline ASA & 1.60 & {$[1.00-2.54]$} & 0.048 \\
\hline BMl & 1.00 & {$[0.94-1.06]$} & 0.876 \\
\hline Hydronephrosis & 1.65 & {$[0.93-2.90]$} & 0.085 \\
\hline
\end{tabular}

$\mathrm{HR}$ - hazard ratio; 95\% Cl - 95\% confidence interval; $\mathrm{RBC}$ - red blood cells count; WBC - white blood cells count; PLT - platelets; HGB - hemoglobin; GFR - glomerular filtration rate; $T$ - tumor stage; $N$ - lymph nodes involvement; ASA - American Society of Anesthesiologists physical status classification system; BMI - body mass index.

Table 4. Results of Cox regression to determine the association with overall survival (OS; multivariate analysis)

\begin{tabular}{|l|c|c|c|}
\multicolumn{1}{|c|}{ Parameter } & HR & $95 \% \mathrm{Cl}$ & p-value \\
\hline Age & 1.04 & {$[1.01-1.07]$} & 0.014 \\
\hline Serum creatinine & 2.16 & {$[1.19-3.93]$} & 0.012 \\
\hline Serum hemoglobin & 0.66 & {$[0.56-0.78]$} & $<0.001$ \\
\hline
\end{tabular}

HR - hazard ratio; 95\% Cl - 95\% confidence interval.

Table 5. Overall survival (OS) by T stage

\begin{tabular}{|l|c|c|c|}
\hline \multicolumn{1}{|c|}{ Survival } & T2 & T3 & T4 \\
\hline One-year & $83.0 \%$ & $67.2 \%$ & $58.6 \%$ \\
\hline Two-year & $72.3 \%$ & $56.8 \%$ & $44.3 \%$ \\
\hline Three-year & $68.0 \%$ & $51.9 \%$ & $39.9 \%$ \\
\hline Four-year & $60.5 \%$ & $40.4 \%$ & $35.9 \%$ \\
\hline
\end{tabular}

analysis (Table 4) performed with the use of Cox proportional hazard regression model, which included selected independent significant variables detected in the univariate analysis (Table 3), it was found that the serum creatinine level was an independent variable significantly associated with OS.

Patients diagnosed with hydronephrosis had significantly higher serum creatinine level $(1.43 \pm 0.45 \mathrm{mg} / \mathrm{dL}$, $\mathrm{p}=0.003)$ compared to patients without hydronephrosis (1.22 \pm 0.35 mg/dL; Fig. 2).

Creatinine serum level was also correlated with $\mathrm{T}$ score ( $<<0.01$; Fig. 3A). Furthermore, the overall survival was associated with T stage ( $<<0.01$; Fig. $3 B$, Table 5$)$. 


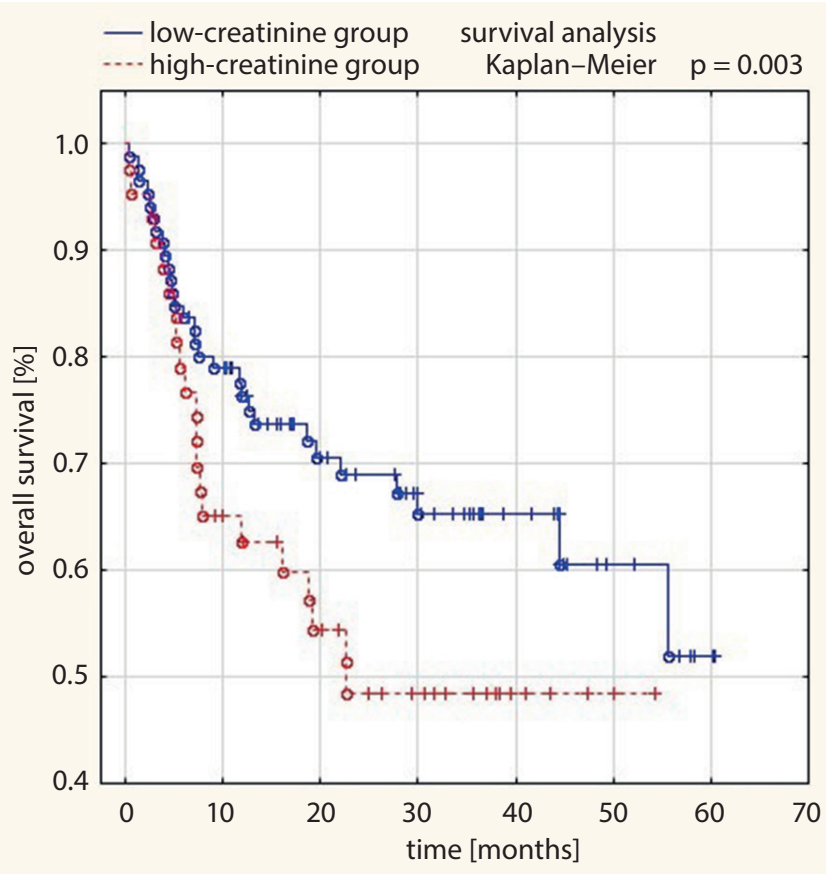

Fig. 2. Comparison of Kaplan-Meier survival curves between patients with or without hydronephrosis
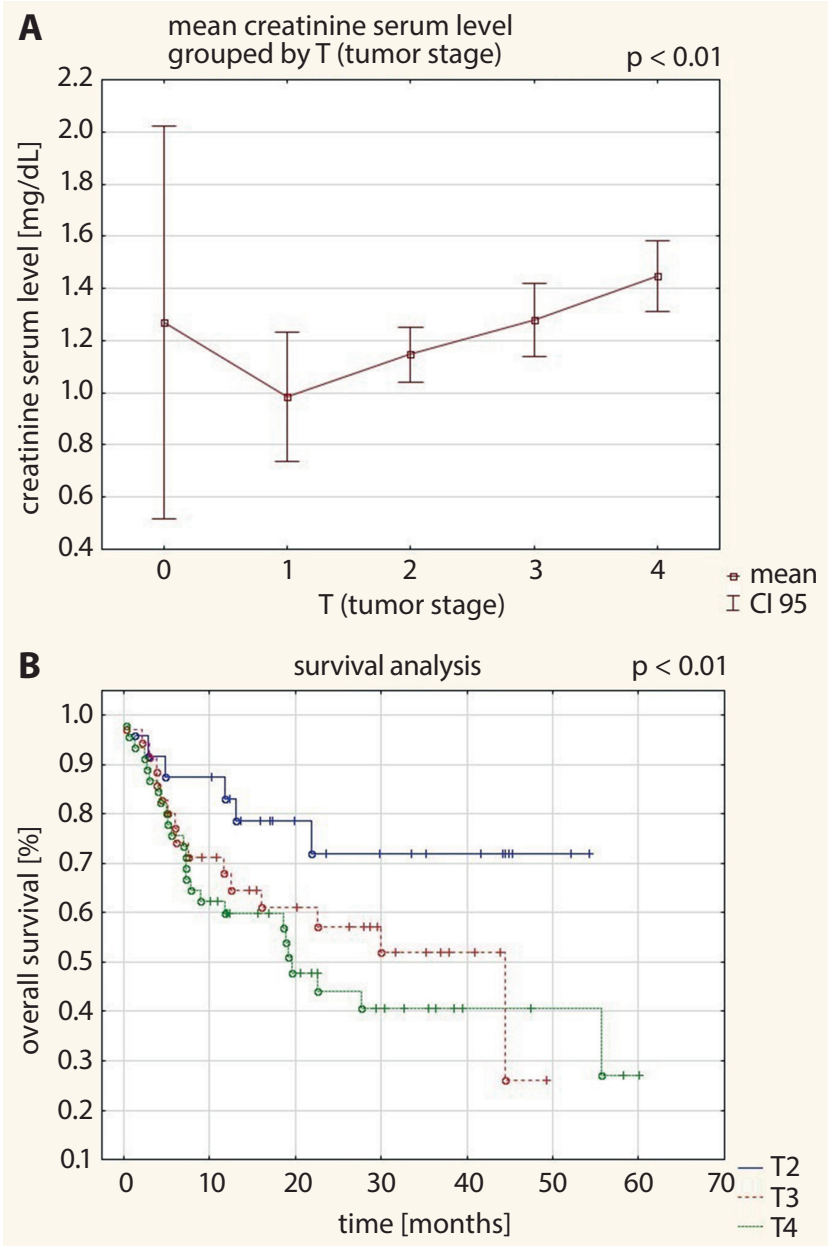

Fig. 3. A. Mean serum creatinine levels grouped by $T$ (tumor stage); B. OS presented as Kaplan-Meier curves grouped by T (tumor stage)

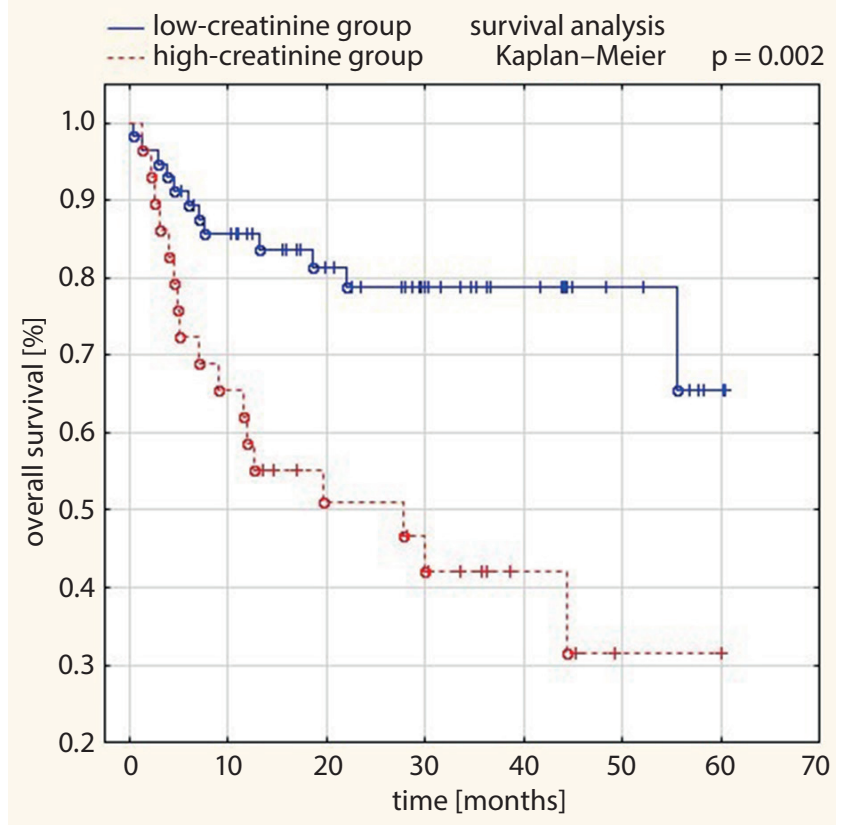

Fig. 4. Comparison of Kaplan-Meier survival curves between low- and high-creatinine groups in patients with hydronephrosis

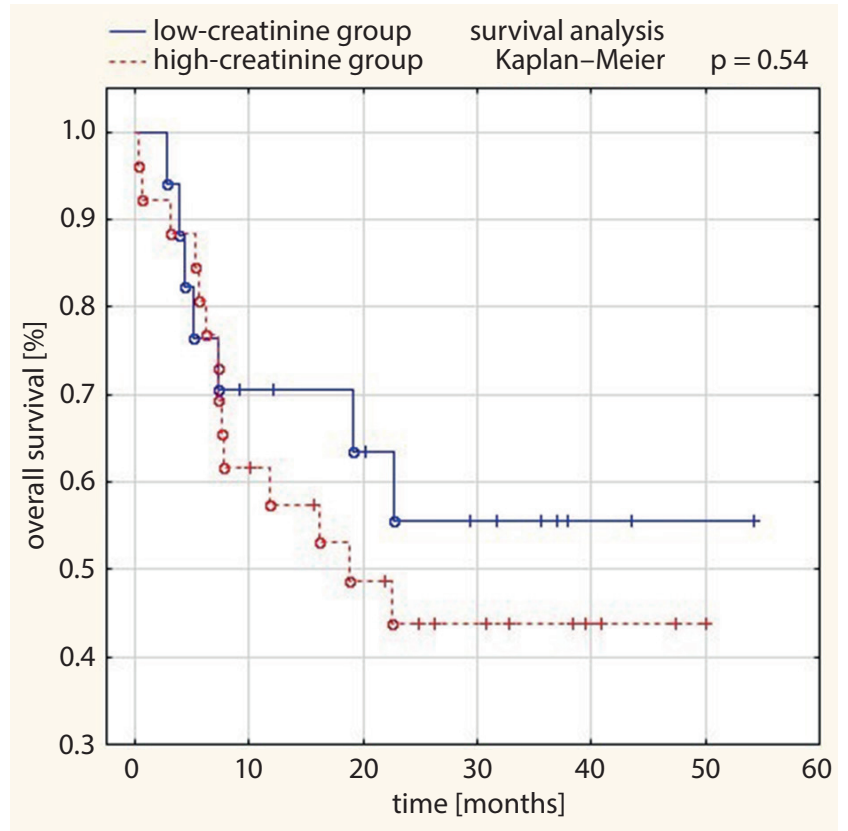

Fig. 5. Comparison of Kaplan-Meier survival curves between low- and high-creatinine groups in patients without hydronephrosis

The OS in patients without hydronephrosis was significantly shorter in those with high serum creatine levels (log-rank test; $\mathrm{p}=0.002$; Fig. 4). There was no statistically significant difference in OS between patient groups of high and low serum creatine level diagnosed with hydronephrosis (Fig. 5; p = 0.54).

The presence of hydronephrosis is related to the severity of the disease. In the case of T3 and T4 disease, dilatation of the renal pelvis was observed in almost half of the patients (Fig. 6). 


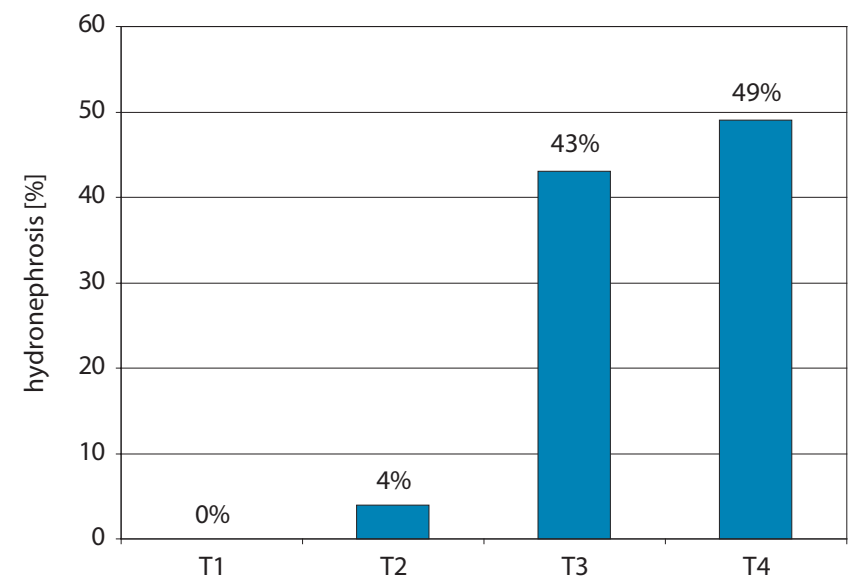

Fig. 6. Percentage of patients with hydronephrosis in different groups of cancer stage

\section{Discussion}

While examining a group of patients undergoing cystectomy, the objective was to find prognostic factors that would allow us to estimate the prognosis for patients with MIBC. The most important prognostic factor is the tumor stage. ${ }^{1}$ Based on the analysis of survival in the study group, it was found that $60.5 \%$ of patients with $\mathrm{T} 2$ cancer survived 4 years, compared to $40.4 \%$ of T3 patients and $35.9 \%$ of T4 patients. Patients with less advanced disease have better results (OS).

It was also shown that preoperative hemoglobin levels are associated with OS after CR, patients with lower hemoglobin levels had a shorter survival time. Anemia reflects the failure of the sick organism. ${ }^{2}$ Advanced bladder cancer often infiltrates the bladder orifices of the ureters, causing hydronephrosis. The obstructed outflow of urine from the kidney leads to a reduction in the filtering capacity of the organ, which results in an increased concentration of creatinine. In the study group, more than $1 / 3$ of patients had hydronephrosis observed during the pre-CR ultrasound examination. Uropathy is a negative prognostic factor, and the probability of four-year survival in a patient with hydronephrosis is lower than in a patient without hydronephrosis (42.3\% compared to 60.6\%). Hydronephrosis is the result of the advancement of the disease. ${ }^{3}$

Survival probability analysis was performed for 2 groups: patients with and without hydronephrosis. In both groups, creatinine levels above the acceptable range were associated with a shorter OS. However, in the case of patients without hydronephrosis, the difference was greater and statistically significant (Fig. 4,5).

Creatinine is a product of creatine metabolism, which is produced in the liver. Creatine is transported to the organs that use the most energy, mainly muscles. Creatine occurs mainly in a form of phosphocreatine (75\%), a highenergy compound that supplies adenosine triphosphate (ATP). About $2 \%$ of the creatine stored in the human body is converted into creatinine every day. Creatinine is the final product excreted in the urine. Elevated blood creatinine levels indicate renal failure. Creatinine concentration depends on the muscle mass, hence the norm for men is higher than for women. In the elderly, decrease in muscle mass is observed, hence a reduced concentration of creatinine. In the case of musculoskeletal dysfunction, a decreased serum creatinine concentration is also observed., ${ }^{4,5}$ Increased creatinine concentration is also observed during intense exercise (that is, during high energy demand). ${ }^{6}$ The correlation between elevated serum creatinine and increased mortality is unclear. Several hypotheses have emerged.

Elevated creatinine levels indicate renal failure, which has a negative effect on the body. ${ }^{7}$ There is evidence of increased mortality in patients with cardiovascular disease (CVD) and renal failure. There are reports of increased mortality in patients with chronic kidney disease (CKD), suffering from diseases other than cardiovascular. ${ }^{8}$

At the end of $20^{\text {th }}$ century, an association of OS with creatinine levels in patients after CR was observed. The serum concentration of $1.5 \mathrm{mg} / \mathrm{dL}$ was adopted as the borderline value. In patients with elevated creatinine levels, the mean OS was 3 years, and in patients with levels below $1.5 \mathrm{mg} / \mathrm{dL}$, it was 8.6 years. ${ }^{9}$

Initially, when looking for a relationship between renal failure and increased mortality, the borderline value of glomerular filtration rate (GFR) was $30 \mathrm{~mL} / \mathrm{min}$, then the limit was shifted to $60 \mathrm{~mL} / \mathrm{min}$. About $37 \%$ of patients are diagnosed with CKD before CR. ${ }^{10} \mathrm{Cao}$ et al. observed that renal failure is associated with more frequent recurrences of urothelial carcinoma. ${ }^{11}$ In cancer patients, the presence of CDK may be due to long-term inflammation that leads to oxidative stress. As a result, renal vessels are damaged. ${ }^{12}$

Yang et al. conducted a study on 9,465 patients with newly diagnosed cancer. A significant relationship between renal failure and OS was found in gynecological and hematological neoplasms. ${ }^{13}$

Vulvar cancer is one of the cancers with proven increased mortality in patients with elevated creatinine levels. One of the hypotheses is that the increased energy demand of aggressive tumors leads to energy being obtained from phosphocreatine. Consequently, this increases the concentration of creatinine in the blood serum. ${ }^{14}$ Neither of these hypotheses explains unambiguously the mechanism of correlating CKD with mortality in patients after RC.

The main limitation of the work was its retrospective nature. The main problem was inconsistent medical documentation and difficult contact with the patients or their families. For this reason, the study group was significantly reduced. A prospective study would help to standardize the collected data. Due to the number of cystectomies performed in 1 hospital, a multicenter study should be performed to increase the study group and thereby the accuracy of the analysis. 


\section{Conclusions}

High mortality after cystectomy prompts the search for prognostic factors. According to the results of our study, serum creatinine has a statistically significant correlation with OS. Creatinine level seems to be a promising parameter. The prognostic value of elevated serum creatinine could be implemented in the nomograms to improve its accuracy. A prospective, multicenter, large cohort study should be conducted, which will allow us to assess cancerspecific survival. Patients with elevated serum creatinine level should be treated with caution. Simple urinary diversion should be considered to minimize the trauma associated with the surgery.

\section{ORCID iDs}

Paweł Hackemer (D) https://orcid.org/0000-0001-7071-2968 Bartosz Małkiewicz (D) https://orcid.org/0000-0002-5933-3753 Fryderyk Menzel (1) https://orcid.org/0000-0002-1150-3151 Aleksandra Drabik (D) https://orcid.org/0000-0001-8525-6387 Krzysztof Tupikowski (D) https://orcid.org/0000-0001-9728-190X Romuald Zdrojowy (D) https://orcid.org/0000-0002-1634-3556

\section{References}

1. Nielsen ME, Mallin K, Weaver MA, et al. Association of hospital volume with conditional 90-day mortality after cystectomy: An analysis of the National Cancer Data Base. BJU Int. 2014;114(1):46-55. doi:10. 1111/bju.12566

2. Bhindi B, Hermanns T, Wei Y, et al. Identification of the best complete blood count-based predictors for bladder cancer outcomes in patients undergoing radical cystectomy. Br J Cancer. 2016;114(2): 207-212. doi:10.1038/bjc.2015.432
3. Kim DS, Cho KS, Lee $\mathrm{YH}, \mathrm{Cho} \mathrm{NH}$, Oh YT, Hong SJ. High-grade hydronephrosis predicts poor outcomes after radical cystectomy in patients with bladder cancer. J Korean Med Sci. 2010;25(3):369-373. doi:10. 3346/jkms.2010.25.3.369

4. Wyss M, Kaddurah-Daouk R. Creatine and creatinine metabolism. Physiol Rev. 2000;80(3):1107-1213. doi:10.1152/physrev.2000.80.3.1107

5. Odden MC, Shlipak MG, Tager IB. Serum creatinine and functional limitation in elderly persons. J Gerontol A Biol Sci Med Sci. 2009;64A(3): 370-376. doi:10.1093/gerona/gln037

6. Mansour SG, Verma G, Pata RW, Martin TG, Perazella MA, Parikh CR. Kidney injury and repair biomarkers in marathon runners. Am J Kidney Dis. 2017;70(2):252-261. doi:10.1053/j.ajkd.2017.01.045

7. Liu M, Li X, Lu L, et al. Cardiovascular disease and its relationship with chronic kidney disease. Eur Rev Med Pharmacol Sci. 2014;18(19): 2918-2926.

8. Fried LF, Katz R, Sarnak MJ, et al. Kidney function as a predictor of noncardiovascular mortality. J Am Soc Nephrol. 2005;16(12):3728-3735. doi:10.1681/ASN.2005040384

9. Thrasher JB, Frazier HA, Robertson JE, Dodge RK, Paulson DF. Clinical variables which serve as predictors of cancer-specific survival among patients treated with radical cystectomy for transitional cell carcinoma of the bladder and prostate. Cancer. 1994;73(6):1708-1715.

10. Hamano I, Hatakeyama S, Iwamura $\mathrm{H}$, et al. Preoperative chronic kidney disease predicts poor oncological outcomes after radical cystectomy in patients with muscle-invasive bladder cancer. Oncotarget. 2017;8(37):61404-61414. doi:10.18632/oncotarget.18248

11. Cao J, Zhao X, Zhong Z, Zhang L, Zhu X, Xu R. Prognostic value of pre-operative renal insufficiency in urothelial carcinoma: A systematic review and meta-analysis. Sci Rep. 2016;6:35214. doi:10.1038/ srep35214

12. Matsumoto A, Nakagawa T, Kanatani A, et al. Preoperative chronic kidney disease is predictive of oncological outcome of radical cystectomy for bladder cancer. World J Urol. 2018;36(2):249-256. doi:10. 1007/s00345-017-2141-2

13. Yang Y, Li HY, Zhou Q, et al. Renal function and all-cause mortality risk among cancer patients. Medicine (Baltimore). 2016;95(20):e3728. doi:10.1097/MD.0000000000003728

14. Schwameis R, Postl M, Bekos C, et al. Prognostic value of serum creatine level in patients with vulvar cancer. Sci Rep. 2019;9(1):11129. doi:10.1038/s41598-019-47560-3 